Revue des patrimoines

\title{
Le «bassin des carènes » et le service technique des constructions navales à Balard : un exemple de cité scientifique à Paris
}

\section{Valérie Gaudard}

\section{OpenEdition}

Journals

Édition électronique

URL : http://journals.openedition.org/insitu/3806

DOI : 10.4000/insitu.3806

ISSN : 1630-7305

Éditeur

Ministère de la Culture

\section{Référence électronique}

Valérie Gaudard, « Le « bassin des carènes » et le service technique des constructions navales à Balard : un exemple de cité scientifique à Paris », In Situ [En ligne], 10 | 2009, mis en ligne le 19 mai 2009, consulté le 21 décembre 2020. URL : http://journals.openedition.org/insitu/3806 ; DOI : https:// doi.org/10.4000/insitu.3806

Ce document a été généré automatiquement le 21 décembre 2020.

\section{(c) $(1) \&$}

In Situ Revues des patrimoines est mis à disposition selon les termes de la licence Creative Commons Attribution - Pas d'Utilisation Commerciale - Pas de Modification 4.0 International. 


\title{
Le «bassin des carènes » et le service technique des constructions navales à Balard: un exemple de cité scientifique à Paris
}

\author{
Valérie Gaudard
}

Entre le boulevard du Général Martial-Valin (autrefois boulevard Victor) et l'avenue de la Porte de Sèvres (Paris, $15^{\mathrm{e}}$ arrondissement) se situe une véritable cité scientifique, sans doute la première de ce type en France par son ancienneté1.

2 La Marine, en l'espèce le Service technique des constructions navales (STCN), aussi dénommé Service technique des constructions et armes navales (STCAN), y a édifié depuis 1904 un ensemble de bâtiments à usage scientifique et technique, destiné à abriter des laboratoires d'étude du comportement des navires en toutes situations.

3 Hormis le premier édifice, l'ensemble présente une grande homogénéité architecturale. De fait, Auguste et Gustave Perret, avec leur agence ou leur entreprise, interviennent sur le site pendant près de trente ans. En nombre de bâtiments, il s'agit de la plus importante de leurs réalisations parisiennes. Elle est par ailleurs bien conservée, ce qui n'est pas le cas d'une autre "cité scientifique » qu'ils édifient également à la demande de l'État, le Centre d'études atomiques (CEA) de Saclay (Essonne). À l'inverse de ce dernier, le site Balard, tributaire des acquisitions laborieuses du terrain et de l'étroitesse des parcelles, a bénéficié d'un plan d'aménagement moins élaboré.

On distingue plusieurs tranches de travaux : au premier bassin achevé en 1906 succèdent, dans les années 1920, la construction de petits bassins et des projets d'ensemble du site, comprenant notamment un laboratoire d'hydrodynamique et un bassin de giration. Les principaux bâtiments, dans la partie est du site, sont élevés à partir de 1932 jusqu'à la veille de la guerre par l'agence Perret frères qui effectue avant et après-guerre des modifications au gré des besoins. Dans les années 1950, enfin, la partie occidentale du site 
est investie, avec la construction de canaux, dont l'un est lié à l'exploitation du bassin de giration.

5 C'est donc un ensemble de bâtiments à l'étroit, aujourd'hui quelque peu ignoré du grand public, que nous nous proposons d'aborder. La principale source d'archives est constituée par le fonds Perret de l'Institut français d'architecture (IFA), où deux mille dessins retracent l'intervention des architectes; mais ces documents n'éclairent pas la totalité de l'histoire du site et des imprécisions subsistent. Les textes restent rares. Cependant, d'importants fonds d'archives n'ont, à notre connaissance, pas été vus; nous nous proposons d'en livrer, pour une partie d'entre eux, une première et rapide lecture. Cette contribution aurait pu être complétée par la consultation des fonds encore en possession de l'affectataire et des visites répétées des lieux, absence qui trace les limites de notre étude.

\section{La longue gestation du premier bassin de traction}

\section{Doter la France d'un bassin : l'obsession de l'ingénieur Bertin}

Lorsque le 9 juillet 1906 est inauguré le premier bassin au boulevard Victor, l'événement, auquel le ministre de la Marine ne manque d'assister, marque l'aboutissement de longues années d'efforts. Instigateur et cheville ouvrière du projet, l'ingénieur, directeur de l'École nationale supérieure du Génie maritime, Louis-Émile Bertin ${ }^{2}$ (1840-1924), en professe le bien-fondé sans succès auprès de son autorité de tutelle depuis plus de dix ans. Son idée était de permettre l'étude du comportement des carènes de navires en laboratoire, ainsi que d'évaluer la puissance des hélices nécessaires à la propulsion. L'application convenable des lois de similitude mécanique ${ }^{3}$ permet de soumettre un modèle réduit, identique au bâtiment à construire, à la traction et d'en déduire, en extrapolant, les prévisions des performances à attendre du navire lui-même.

7 Pour ce faire, un bassin d'essai produisant des conditions d'observation toujours identiques était nécessaire.

\section{Les exemples étrangers}

Dès 1870, Bertin avait présenté à l'Académie des Sciences un mémoire sur la résistance des carènes dans le roulis. Il est au fait des réalisations étrangères : le monde occidental est en effet déjà bien doté de bassins de ce type lorsque celui de Balard voit le jour. Cette même année 1870 est construit le premier bassin à Torquay (Grande-Bretagne). L'Italie, à La Spezia, l'Allemagne à Bremerhaven et Berlin, la Russie à Saint-Pétersbourg, ainsi que les États-Unis à Washington, possèdent des bassins d'essais ${ }^{4}$. Les exemples étrangers sont profitables au projet français puisqu'ils permettent de l'optimiser: les dimensions du bassin sont établies par Bertin après une mission de la jeune Section technique des constructions navales ${ }^{5}$ en Angleterre, à Bremerhaven et au vu des données recueillies à La Spezia et à Washington. Le bassin privé de Bremerhaven, qui ne possèderait pas les dimensions suffisantes pour garantir la fiabilité des résultats, sert de repoussoir, cependant que son organisation associant canal et ateliers est au contraire plébiscitée. 


\section{Les obstacles topographiques et financiers}

9 Si la nécessité de doter la France de cet instrument d'expérimentation semble acquise à la toute fin du XIX ${ }^{e}$ siècle, les obstacles, notamment topographiques et financiers, retardent sa construction. Ces deux contraintes pesèrent sur l'histoire du site Balard, jusqu'à nos jours.

Après de nombreuses recherches ${ }^{6}$, un projet d'implantation à l'emplacement du bastion numéro 69 de l'enceinte de Thiers voit le jour en 1902. Plusieurs raisons conduisent à ce choix. La Défense nationale possède depuis peu un terrain voisin à Issy-les-Moulineaux, champ de manœuvres acquis après échange du Champ de Mars avec la Ville de Paris en 1890. Par ailleurs, la présence d'un sol à niveau du boulevard Victor et d'un remblai estimé de $2 \mathrm{~m}$ constituant le terrain d'assiette du futur bassin entraînerait peu de travaux de terrassements et de fondations ${ }^{7}$. Enfin la nappe phréatique, à $4 \mathrm{~m}$ de profondeur, se trouverait au niveau du radier du bassin. Les sujétions existent mais sont considérées comme mineures. Le terrain disponible est tout juste suffisant: situé dans le terre-plein du rempart, il est limité au nord par l'ancienne route militaire formée par le boulevard Victor et les flancs et les faces du bastion sur les autres côtés. La réalisation nécessitera de dévier la route militaire.

11 En tout état de cause, le projet de bassin fut dessiné sans implantation précise. Le choix du bastion numéro 69, proposé en septembre 1902 au ministre, intervient après les premières ébauches de l'édifice réalisées peut-être vers 1900, du moins au printemps de la même année. Il faut encore patienter jusqu'au début de l'année 1904 pour que la cession soit effective ${ }^{8}$.

\section{Des projets revus à la baisse}

Le vote par le Parlement, le 31 mars 1902, du financement de la construction, permet à la section technique de fournir aussitôt les projets détaillés qu'elle tenait prêts. Dès l'origine, le bâtiment se compose de deux entités formées, d'une part, par les ateliers et les bureaux, d'autre part, à leur extrémité, par le bassin proprement dit en forme de canal. Il est remarquable que l'on se préoccupe de la qualité esthétique de l'édifice. Son caractère scientifique ne doit pas être un prétexte à faire l'impasse sur certaines qualités demandées à toute architecture, quelle que soit sa nature. Le boulevard fréquenté, la visite escomptée de nombreux étrangers engagent le maitre d'œuvre: il y a lieu de " concilier dans une juste mesure le caractère de simplicité industrielle que doit présenter un laboratoire d'expériences avec les convenances architecturales si modestes soient-elles qu'il est nécessaire d'assurer aux édifices publics en façade sur la rue dans une ville telle que Paris $»^{9}$. Aussi les premiers dessins proposés cherchent-ils à rompre la monotonie de la longue façade par le jeu des matériaux et la volumétrie.

Les dimensions du bâtiment connaissent de nombreuses variations, Bertin tentant de concilier prospective scientifique et considérations financières. Alors que le projet est prêt depuis un an, le ministre le rejette en mars 1903 au motif d'une trop grande dépense. La largeur du bâtiment recouvrant le canal est ramenée de 15 à $12 \mathrm{~m}$, dimension définitive. L'ingénieur accepte de supprimer trois travées au bassin, réduisant ce dernier de $160 \mathrm{~m}$ à $146 \mathrm{~m}$ puis $140 \mathrm{~m}$. Mais il insiste sur la nécessité de réserver le terrain pour l'agrandissement du canal quand les fonds seront alloués et quand l'expérience et les 
progrès techniques auront montré la nécessité d'un bassin plus long. Au final, le bassin a une longueur utile de $135 \mathrm{~m}$, ce qui le place en quatrième rang des bassins mondiaux, après Berlin $(146 \mathrm{~m})$, Bremerhaven et La Spezia ${ }^{10}$. Réduits, les ateliers subissent le même sort.

\section{Le programme et sa réalisation : le choix du ciment armé} travaux de terrassement et de maçonnerie du bassin qui sont adjugés à l'entrepreneur Ferdinand Pradeau ${ }^{11}$. Le concours pour la construction du bâtiment surmontant le bassin et des ateliers est ouvert en février de l'année suivante. Quatre mois plus tard, le 16 juin 1904, le marché est adjugé à l'entreprise Chassin et fils ${ }^{12}$.

15 Le programme prévoit la construction d'un bâtiment de 160,90 $\mathrm{m}$ de long et $12 \mathrm{~m}$ de large, contenant un bassin diminué pour le service de $2,50 \mathrm{~m}$ en longueur et $2 \mathrm{~m}$ en largeur, et d'une profondeur de $4 \mathrm{~m}$. Sa forme de cuvette à radier circulaire est réputée plus économique. Avec ses ateliers, le bâtiment, d'un seul niveau, totalise une longueur de $225 \mathrm{~m}$. Le programme prévoit une couverture en charpente métallique. Mais il est remarquable que l'entreprise Chassin remporte le marché en proposant une charpente en ciment armé ${ }^{13}$. La préférence donnée au ciment par l'État est en partie d'ordre financier, le métal coûte plus cher que le béton et un nombre inférieur de fermes fut nécessaire à la réalisation de la charpente en ciment armé : Chassin propose en effet une ferme tous les $10 \mathrm{~m}$ au-dessus du bassin ( $7 \mathrm{~m}$ dans l'atelier) lorsque ses concurrents prévoient un espacement entre 5 et $7 \mathrm{~m}^{14}$ ! Justifiant son choix, la Marine reconnaît par ailleurs un développement de l'usage du ciment et reprend à son compte les arguments avancés par l'entrepreneur : incombustibilité du matériau, tant vantée par les entrepreneurs en béton armé, capacité à conserver la chaleur et réduire ainsi les coûts de chauffage, faible nécessité d'entretien, contrairement à une charpente métallique susceptible de rouiller en atmosphère humide. La mise en œuvre semble cependant plus longue puisque le délai demandé par Chassin est de six mois au lieu de cinq pour ses concurrents.

ciment armé est coulé sur place dans des coffrages fabriqués au préalable. La facture laisse penser que la totalité de chaque ferme était coulée d'une pièce, leurs éléments semblant solidaires les uns des autres. Comparativement au métal, même si Chassin en reste proche, l'utilisation du béton permet aussi d'alléger sensiblement d'un certain nombre d'aisseliers les fermes triangulées, reliées entre elles par des sablières hautes. L'espace entre les poteaux est rempli de briques. Sur le côté sud du hall est établie une passerelle d'observation. Un lanterneau vitré, aujourd'hui occulté, apporte la lumière nécessaire aux expériences...et conduit à une prolifération de bactéries dans l'eau du bassin. Ce bâtiment est un remarquable témoignage d'une charpente en ciment armé, par la relative précocité du procédé mais également la rareté des exemples conservés et attribués. exprimé dans le premier projet succède, dans le programme du concours, la prescription d'édifier « des bâtiments de construction durable mais conçus dans le style des ateliers les plus simples $»^{15}$.

18 Comme nombre de ses concurrents, l'entreprise Chassin, fondée en 1867, est spécialisée dans les travaux de ciment armé pour l'exploitation du gaz et de l'eau ${ }^{16}$. Henri Chassin dépose plusieurs brevets de perfectionnement à la construction en ciment armé, 
notamment en 1897 et $1898^{17}$. Souvent primée, l'entreprise rassure la Marine par ses sérieuses références. On peut penser que sa réalisation boulevard Victor donne satisfaction puisque le ministère de la Guerre lui commande en 1910 un bâtiment composé d'un hangar central, de bureaux et d'un atelier pour l'établissement aéronautique de Vincennes ${ }^{18}$.

\section{Une chaîne opératoire complète}

L'ensemble des opérations nécessaires à la fabrication des maquettes est réalisé sur place, dans les ateliers situés à une extrémité du canal. Le modèle y est dessiné, le moule réalisé en bois, la paraffine coulée dans le moule. Une imposante machine à tailler les modèles permet d'affiner la forme, parachevée à la main sur un banc à ébarber, polir et vérifier le modèle. L'archivage des données et dessins et la conservation des modèles de carènes et d'hélices font partie de l'activité des ingénieurs, tandis que la salle consacrée à la photographie, présente dans les premiers projets, disparaît par la suite, sans doute par souci d'économie. Une fois réalisées, les maquettes sont transportées au moyen de ponts roulants dans l'atelier et jusqu'au bassin. Glissant sur des rails posés le long des arêtes de ce dernier, circule, au-dessus du bassin, une plate-forme où prennent place le personnel et leurs instruments. La maquette du bateau, entrainée par la plate-forme dont la vitesse peut atteindre 5 mètres par seconde, évolue sur l'eau du canal. Les conditions reproduites sont celles de "l'eau infinie ", c'est-à-dire sans effet de fond ni de parois. Le bassin est alimenté par l'eau de la ville.

Il subit sans dommage la crue de 1910. Aux usages militaires furent rapidement ajoutés des essais pour la marine marchande à titre onéreux. Dès 1908 semble-t-il, la compagnie générale transatlantique fait flotter ses modèles de paquebots sur l'eau du bassin. La défiance des armateurs dans cette expérimentation rend ces essais commerciaux cependant trop rares. L'activité est tournée vers l'essai de navires en projet, bien plus que, faute de personnel, la recherche fondamentale.

\section{Une cité scientifique des frères Perret}

\section{Un projet d'envergure}

21 Au lendemain du premier conflit mondial, l'enseignement tiré des batailles navales incite à étendre le champ d'activité de la section technique. L'étude de questions nouvelles, telles que la manœuvrabilité des navires et leur tenue à la mer, entraîne un accroissement des besoins. C'est le directeur du bassin de 1920 à 1941, l'ingénieur en chef du génie maritime Barrillon, qui préside aux choix et donne au site son orientation. Au gré des cessions de terrains, en s'adjoignant l'une des meilleures agences d'architecture nationales, il donne au lieu son actuelle physionomie.

Malgré la vétusté du premier édifice dit bassin numéro 1 , le choix est fait de porter l'accent non sur la création d'un nouveau bassin de traction ou l'extension du premier comme l'avait envisagé Bertin mais sur les laboratoires. À la fin des années vingt, quelques aménagements sont tout d'abord effectués, telle l'édification d'un petit canal, de petites soufflerie et cuve, préalables souhaités au projet de constructions d'importance. Sans doute l'idée d'élever un bâtiment pour le personnel de conception et d'exécution, un laboratoire d'hydrodynamique, même modeste, et un bassin de giration est-elle mûre au 
début des années vingt. Malgré les $65 \mathrm{~m}$ de diamètre du bassin, sa construction est envisagée dès avant les cessions complètes des terrains du bastion à la Marine ${ }^{19}$. La loi de démantèlement des fortifications de Paris (1919) ouvrait en effet des perspectives aux occupants des lieux mais leur patience fut mise à rude épreuve ${ }^{20}$.

L'agence Perret frères ou l'entreprise édifient, de 1928 jusqu'à la mort d'Auguste en 1956, un ensemble de constructions à vocation scientifique et technique. Si plusieurs d'entre elles sont reprises, étendues, modifiées selon l'usage au cours de l'activité des architectes, le dessin, identique, tout comme le mobilier urbain également dessiné par Perret, confère cependant au site son homogénéité.

\section{Les bureaux du STCN donnent le ton}

Le personnel du service technique des constructions navales (STCN), alors logé dans les combles du ministère de la Marine rue Royale, est promis à plus d'efficacité au contact même des locaux d'expérimentation. À l'angle du boulevard Victor et de l'avenue de la Porte de Sèvres, le bâtiment du STCN est édifié en plusieurs temps. Aux plans réalisés à partir de décembre 1928 succède le permis de construire, déposé le 19 avril 1929 et accepté en août ${ }^{21}$. Au fil des projets, Perret recherche une plus grande unité du bâtiment : il abandonne l'idée de pavillon à la jonction du corps central et des deux ailes en retour, puis le pan coupé au profit d'un angle arrondi et l'élévation d'un troisième étage à ces mêmes angles. À la volonté de distinguer, de façon classique, l'articulation entre le corps principal et les ailes succède la recherche plus moderne d'un rythme intrinsèque, unifié sur l'ensemble du bâtiment. Elle permet à la fois de jouer sur le plan masse pour fondre toujours davantage l'édifice, qui épouse les courbes des voies, dans son environnement alors presque vierge de construction, et de souligner les articulations non plus en plan mais en élévation, mettant en exergue l'architectonique.

Au-delà des qualités plastiques indéniables de cet immeuble, les critiques louent la science technique que l'architecte met d'ailleurs lui-même en avant dans les revues spécialisées: correction des erreurs optiques de la façade pour éviter l'impression "d'affaissement milieu », façades et toitures isothermes à triple paroi enserrant deux matelas d'air qui offrent également une protection contre les bombes incendiaires, chauffage à air pulsé. Surtout, l'ossature en béton armé avec son remplissage de panneaux de béton préfabriqués permet une grande modularité tant à l'extérieur qu'à l'intérieur: les ouvertures en façade, au dessin particulièrement soigné, peuvent être facilement modifiées tout comme la distribution, autorisant une grande liberté du cloisonnement ${ }^{22}$.

La circulation verticale, au centre du corps principal et à l'angle des ailes, donne lieu à un traitement de la façade postérieure avec claustras. À chaque étage, les bureaux sont distribués de part et d'autre d'un long couloir central, à l'exception du deuxième étage, très remarqué, avec sa vaste salle de dessin de $1600 \mathrm{~m}^{2}$ (« unique au monde $»^{23}$ ) largement éclairée sauf au sud.

Le bâtiment est modifié à plusieurs reprises, transformant quelque peu le dessin initial. Fonctionnant comme un attique, la surélévation, autorisée en décembre 1936, est prévue dès l'origine et les fondations sont calculées en conséquence. Dix ans plus tard, l'extension de l'aile est le long de l'avenue est entreprise, sur les dessins de l'architecte conseil du STCN Camille Marion ${ }^{24}$. Le STCN acquiert sa physionomie actuelle après une ultime surélévation des ailes est et ouest projetée en 1953. Le Conseil général des 
bâtiments de France exige une implantation très en retrait, à l'image de la première surélévation réalisée par Perret ${ }^{25}$.

\section{Les laboratoires, premiers compléments à l'extension du champ d'étude scientifique}

Le premier bâtiment construit par les Perret donne le ton général à l'ensemble. Dans la foulée vont s'élever des constructions étudiées simultanément à celle du STCN : tout d'abord un laboratoire de résistance des matériaux puis un laboratoire d'hydrodynamique, des bureaux du bassin, tous élevés au début des années trente et pendant ou après-guerre ainsi qu'un bassin de giration (élevé en 1938 et couvert en 1951). Dans les années cinquante, un canal dit de Suez puis un canal associé au bassin de giration, un atelier de dessin et des bureaux complètent l'ensemble. Enfin, certaines parties des laboratoires sont transformées en bureaux ces dernières années.

L'ensemble de ces installations consacra le site Balard comme l'un des plus importants lieux d'étude de l'hydrodynamique au monde, alliant la recherche de la puissance propulsive à celle de bonnes qualités nautiques et évolutives.

De facture similaire, les deux laboratoires (de résistance des matériaux et d'hydrodynamique) sont également parents du bâtiment du STCN. Peter Collins montre comment Perret place sur le même plan et accorde égale valeur aux bureaux et aux bâtiments techniques. Distants de quelques mètres et visibles d'un seul coup d'œil, ils bénéficient du même mode constructif : ossature béton dont la fonction architectonique est soulignée, remplissage en dalles de béton aux agrégats soignés. Le parti choisi confirme sa modularité: sous la forte corniche, les travées aveugles peuvent être ouvertes par des châssis au gré des besoins. Comme pour le bassin numéro 1, l'éclairage choisi est zénithal, par le biais de sheds à voûte parabolique brevetés par les Perret. Collins a souligné la qualité de leur dessin particulier, couronnant l'édifice d'une série d'arcs ne rompant pas «le calme et l'équilibre de la composition ${ }^{26}$ ». Élevés d'un seul étage, les deux bâtiments se présentent comme une succession de nefs.

31 Le laboratoire d'hydrodynamique abrita une cuve à houle (mise en service en 1935), complétée en 1942 par une petite cuve de tangage, un grand canal à mouvement d'eau remplacé par un tunnel à cavitation en 1948 dont la construction fut décidée en 1939. Désaffectée mais toujours en place, la cuve à houle était la première du genre à sa création. Ce bassin de $30 \mathrm{~m}$ sur 7, d'une profondeur de $2,40 \mathrm{~m}$, permettait des essais de tangage et roulis au point fixe sur des modèles de 4 à $6 \mathrm{~m}$. Un batteur à houle, volet placé à une extrémité du canal, générait une houle régulière pour calculer la résistance des coques.

La cavitation est un phénomène qui se produit dans un liquide au contact des corps animés d'une grande vitesse ; une dépression locale très importante peut aller jusqu'à la création de vapeur d'eau. Elle entrâne des pertes de rendement, une érosion des hélices, $\mathrm{du}$ bruit et des vibrations quand les bulles implosent, que l'ouverture du tunnel de cavitation permit d'étudier. Ses dimensions exceptionnelles en faisaient le plus grand d'Europe, concurrençant celui de Hambourg où les hélices du paquebot Normandie, fleuron de la marine commerciale nationale, avaient été essayées! Cette installation n'est plus en place, mais les dessins permettent de mesurer le gigantisme du tunnel 
comprenant la veine d'essai permettant de recevoir des hélices de $60 \mathrm{~cm}$ de diamètre et la pompe qui mettait l'eau en mouvement.

\section{Le bassin de giration, une première mondiale} exceptionnel est, à notre connaissance, peu documenté dans les archives publiques. Réclamé dès 1924, il ne fut entrepris qu'en 1939. En 1942, lors de sa mise en service, il était unique au monde tant par ses dimensions que sa conception et le resta pendant quinze ans. Sa vocation est d'éprouver les qualités manœuvrières des modèles. Il est constitué d'un plan d'eau circulaire de $65 \mathrm{~m}$ de diamètre et de $5 \mathrm{~m}$ de profondeur. Un bras tournant, dont une extrémité est fixée sur un pivot au centre du bassin et dont l'autre circule dans un rail sur le pourtour, permet d'entraîner les maquettes pour des essais de giration forcée, dans un plan tant horizontal que vertical pour les sous-marins. Sur le bras est fixé un chariot, mobile, qui peut se placer à n'importe quel rayon. Perret fournit trois projets, datés du 13 juin 1938. L'idée directrice demeure semblable: un «manège » de $79 \mathrm{~m}$ de diamètre comprenant un bassin et sa piste, cette dernière, ainsi qu'une partie du bassin, étant couverte par un auvent en béton en porte-à-faux. Des fondations en béton triangulées supportent la piste et son auvent, une poutre soutenant la largeur totale de celle-ci à intervalles réguliers. Seul le traitement de l'étanchéité de la cuve fait l'objet de diverses propositions. L'ouverture d'un diamètre de $53 \mathrm{~m}$ au-dessus de l'eau exposant les essais aux intempéries, dès 1948 on étudie la possibilité de couvrir l'édifice.

S. Texier et G.-A. Langlois ont souligné la difficulté de connaître la part exacte prise par les Perret dans la réalisation du bassin, aucun dessin d'exécution n'étant conservé dans le fonds Perret aux Archives nationales. Il semble que l'on puisse attribuer avec quelque certitude la paternité du bassin à Camille Marion (1882-1964). Architecte de la Marine militaire (constructions navales) depuis 1926, il conduit et surveille les travaux exécutés par les frères Perret pour le STCN. Ces états de service mentionnent le bassin de giration au titre des travaux exécutés sur le site et le distinguent de l'ensemble de ses interventions de surveillance. Par ailleurs, une coupe présentant le bassin avec sa couverture métallique indique quelques différences notables: la piste ne repose que sur des poutres, sans récupération dans le sol de la poussée exercée par l'auvent. Pour en diminuer les effets, le demi-arc formé par l'auvent présente un profil plus aplati. Dans la limite imposée par le caractère technique du bâtiment, Marion s'inspire indéniablement des projets proposés par Perret ${ }^{27}$.

Ce familier de l'agence Perret doit faire preuve de ténacité pour obtenir la signature d'une convention avec la Marine pour l'étude de l'avant-projet de la couverture du manège, réalisée en charpente métallique, et des projets de concours. Le travail envisagé " relevant plus de la technique de la construction immobilière que de l'art architectural ", le caractère industriel et l'aspect artistique «négligeable » de cet immeuble incitaient la Marine à faire l'économie d'un architecte pour le projet, jugeant suffisants les plans de l'entrepreneur. L'entreprise Rouzaud remporte le marché en $1951^{28}$.

\section{Les dernières créations}

Dans la foulée sont entrepris les derniers grands chantiers du site. Les laboratoires étant construits, les projets anciens concernant les bassins de traction sont repris après-guerre. 
Par marché passé en 1949 avec l'entreprise Perret ${ }^{29}$, le STCN entreprend un deuxième bassin : progressivement prolongé au gré des cessions de terrain, ce dernier atteint $155 \mathrm{~m}$ de long sur $8 \mathrm{~m}$ de large à son achèvement en 1955. Ce canal dit «de Suez» doit reconstituer en laboratoire les conditions de navigation en eaux limitées, notamment celles des gros pétroliers. La pose de berges métalliques permet de modifier la section du bassin pour reproduire le profil de canaux tels celui de Suez. L'application est donc directement à l'usage de la marine marchande.

Enfin, en 1951, simultanément à la couverture du bassin de giration, est entreprise l'amorce d'un troisième et dernier bassin de traction. Appelé canal associé, il communique par une darse de $8 \mathrm{~m}$ de large avec le bassin circulaire dans le but de permettre le lancement d'un modèle à grande vitesse. Ses $220 \mathrm{~m}$ en font le plus long du site, même après l'abandon d'un projet de prolongement jusqu'à la Seine. La plupart des vaisseaux modernes de la flotte française, tant militaire (le Charles-De-Gaulle) que civile, bateaux de pêche et de course (le Pen Duick III de Tabarly) compris, a connu ce bassin, ainsi que les modèles de plates-formes de forage offshore. Ses dimensions ont permis l'essai de navires rapides (les ferries rapides). Contigus, les deux derniers bassins de traction communiquent sur la longueur qui leur est commune.

Le site est complété en 1955 par des ateliers et en 1958 par des bureaux le long du bassin n $\circ 1$.

Par leur antériorité et le type de recherches qui y sont menées, les bâtiments de la Marine à Balard sont à rapprocher de la soufflerie créée en 1912 rue Boileau (Paris, 16 arrondissement) par Gustave Eiffel pour l'étude de l'aérodynamique. La comparaison fait d'autant mieux apparaitre les différences: la dimension du site (4 hectares), son antériorité et la continuité de son occupation (depuis 1904), son enrichissement constant par de nouvelles installations, la taille de ces dernières. Par ailleurs, les installations du STCN forment un site scientifique où est décliné l'ensemble de la chaîne opératoire, depuis les bureaux de conception jusqu'aux laboratoires en passant par la réalisation de l'objet d'expériences. La création d'une école d'application du génie maritime a été un temps envisagée : le site aurait été complété par un volet d'enseignement des savoirs, pour la formation sur place du personnel technique. Dès 1904, le STCN s'est également doté des techniques les plus modernes de conservation de la mémoire des expériences, des résultats et des objets. Enfin, nous avons déjà souligné la cohérence architecturale exceptionnelle de ce site à l'échelle d'un quartier de ville densément bâti. Même si l'intervention des Perret est à réévaluer après-guerre, l'ensemble porte leur marque. Avec l'immeuble du STCN, Perret met en œuvre ses conceptions sur la fonctionnalité de la salle de travail, ajuste les cheminements, généralise le confort et propose une architecture administrative de qualité, digne de sa fonction nationale. De ce fait, il mène à terme sa recherche sur la mise en œuvre du béton armé, en proposant des panneaux de remplissage de béton préfabriqué. Surtout, soucieux de cohérence de l'ensemble des bâtiments, il traite sur un pied d'égalité l'immeuble de bureaux et le laboratoire et accorde la fonctionnalité avec l'esthétique, trop souvent oubliée quand il s'agit d'architecture à destination scientifique ou industrielle. À l'économie du premier bassin répond la monumentalité des bâtiments de Perret: «l'architecture industrielle est ici portée au degré le plus élevé de l'art $»^{30}$.

Par leur taille, certaines installations sont à rapprocher de la big science de l'après-guerre. Le site soulève une fois encore la question de la préservation des installations techniques ou scientifiques lorsqu'elles sont obsolètes. Si le bassin de giration et son canal associé 
sont encore utilisés pour des essais, les laboratoires ont perdu leur usage d'origine ; les bassins $\mathrm{n}^{\circ} 1$, à la charpente si intéressante, et $\mathrm{n}^{\circ} 2$, fermés, ne sont plus en eau. Depuis 1996, les études s'opèrent sur les 20 hectares du site de Val-de-Reuil ${ }^{31}$ (Seine-Maritime), mais le bassin de giration reste toujours un unicum $^{32}$. La communauté scientifique commence à être sensibilisée à cette question de la préservation de la mémoire, notamment du patrimoine instrumental mais également architectural ${ }^{33}$. Aujourd'hui, seul le bâtiment du STCN fait l'objet d'une protection au titre des Monuments historiques, depuis déjà de nombreuses années. L'inscription de 1965 reconnaît, moins de dix ans après la mort d'Auguste Perret, toute la valeur de cet architecte tant sollicité par l'État pour ses opérations les plus prestigieuses, ici l'une de ses toutes premières collaborations, du Mobilier national au Centre d'études atomiques de Saclay en passant par la reconstruction du Havre, au même titre que sont protégées cette année-là, sous l'impulsion d'André Malraux, les œuvres des architectes majeurs du siècle, Guimard et Le Corbusier. Le chemin est un peu plus long pour reconnaittre à l'architecture scientifique une place à part entière, celle de témoignage de la recherche de l'homme dans sa compréhension de ce qui l'environne.

\section{NOTES}

1. La dénomination du lieu est multiple. L'ensemble du site de la Défense situé entre la Seine et le parc des expositions est communément appelé "Cité de l'Air», divisé en parcelle est où se situent les services de l'Air et parcelle ouest pour les services de la Marine. Les services de la Marine ne bénéficient donc pas d'appellation claire : STCN (devenu STCAN) ou Bassin d'essai des carènes (BEC) voire Bassin désignent au sens large l'ensemble des bâtiments situés sur la parcelle $C$ (parcelle ouest) du site Balard du ministère de la Défense.

2. L'œuvre de Bertin dans le domaine maritime est immense. On lui doit de nombreux ouvrages et des inventions qui lui valurent d'être sollicité par l'empereur du Japon pour réorganiser la marine nationale. Son buste orne la baie de Tokyo.

3. Elles furent énoncées en 1852 par l'ingénieur français Reech, professeur de Bertin à l'École nationale supérieure du Génie maritime dont il fut également directeur. Dans l'ignorance des découvertes françaises, l'Anglais William Froude les formula également, en 1869, avec vérification immédiate dans son bassin de Torquay.

4. D'autres bassins existent, cités dans : PIAUD, L. Constructions navales, le bassin d'expériences de la Marine française à Paris. Le Génie civil, 8 septembre $1906, \mathrm{n}^{\circ} 1265$, tome XLIX- $\mathrm{n}^{\circ} 19$, p. 289-293. Également très bonnes explications sur le fonctionnement des machines et instruments du bassin de Paris.

5. Créée en 1895, elle a à sa tête l'ingénieur Bertin l'année suivante. Installée à Paris rue Royale, elle emporte la construction d'un bassin d'essai dans la capitale aux dépens des arsenaux. Sa mission est double: dresser les plans de tous les navires à construire et de leurs appareils propulsifs, contrôler les études de détail qui restent à la charge des ports.

6. En 1899, la Marine sollicite la cession par la Ville de Paris d'un terrain de 5000 à $6000 \mathrm{~m}^{2}$ au bois de Vincennes, demande restée lettre morte. Villeneuve-l'Estang près de Garches en 1897, Juvisy-sur-Orge en 1900, enfin le bastion numéro 30 de l'enceinte de Thiers sont envisagés (SHD/ $\mathrm{DM}, \mathrm{DD}^{2}$ 2228). Max Aucher cite une dépêche ministérielle de 1894 approuvant l'installation à 
Guérigny (Nièvre), dans 100 ans d'histoire du bassin d'essais des carènes. [s.l.] : DGA, Ministère de la Défense, [2006], p. 101.

7. Le programme du concours ramène l'estimation de l'épaisseur du remblai à 1,25 $\mathrm{m}$.

8. Une instruction mixte réunit le ministère de la Guerre, celui de la Marine et la Ville de Paris pour fixer les conditions de la cession des terrains, à titre gracieux. Le terre-plein est concédé par la Guerre à la Marine, sous réserve de rétablir les lieux dans leur état d'origine en cas de conflit. Le ministre de la Marine l'approuve en décembre 1903. Les problèmes d'adjudication par la Ville traînent jusqu'en février 1904.

9. SHD/DM, DD 2228, note du 30 août 1902.

10. PIAUD, L. Constructions navales, le bassin d'expériences de la Marine française à Paris. Le Génie civil, 8 septembre 1906, $\mathrm{n}^{\circ} 1265$, tome XLIX-n ${ }^{\circ} 19$.

11. Entreprise de travaux publics Ferdinand Pradeau, 8 rue Paul-Louis Courier à Paris. Les longues formalités de l'instruction mixte sont cause de la notification tardive du marché à l'entrepreneur, le 8 mars 1904, soit 6 mois après l'obtention du marché. Les travaux débutent à cette date et sont achevés fin juin. Leur qualité est louée par les ingénieurs de la Marine.

12. Henri Chassin et Fils, entreprise de travaux publics et particuliers, spécialité de travaux de ciment, 151, rue de Bagnolet, 8 rue des Lyannes, Paris (20e arrondissement).

13. On ne compte que quatre entreprises soumissionnaires. Seul Chassin propose le ciment armé. 14. Le texte de : PIAUD, L. Constructions navales, le bassin d'expériences de la Marine française à Paris. Le Génie civil, 8 septembre $1906, n^{\circ} 1265$, tome XLIX- ${ }^{\circ} 19$, comporte sans doute une erreur lorsqu'il mentionne une ferme tous les $19,96 \mathrm{~m}$. Il faut lire 9,96 m.

15. SHD/DM, DD2 2229.

16. La base Mérimée mentionne trois réalisations de Chassin : deux réservoirs (Montreuil, SeineSaint-Denis, 1er quart du 20e siècle, IA93000139 ; Athis-Mons, Essonne, 1907, IA91000505) et un pont (Fontenay-le-Comte, Vendée, 1911, IA85000483)

17. BOSC, Jean-Louis (et al.). Joseph Monier et la naissance du ciment armé. Paris : éditions du Linteau, 2001.

18. HODEIR, Marcellin. Vincennes, berceau de l'aéronautique militaire. Revue historique des armées, 2008, n² 251. URL : http://rha.revues.org//index318.html [05/08/08].

19. Fonds Perret, AN/IFA, 535 AP 27/300 document CNAM 28.8.60. S. Texier et G.-A. Langlois font remonter le projet de construction à 1924. TEXIER, Simon, LANGLOIS, Gilles-Antoine. Les bassins d'essais de la Marine. Le XV arrondissement. Voir aussi AUCHER, Max. 100 ans d'histoire du bassin d'essais des carènes. [s.l.] : DGA, Ministère de la Défense, [2006].

20. Si le décret du 29 juin 1928 remet à la Marine les terrains situés le long du boulevard entre le bassin des carènes et l'avenue de la Porte de Sèvres, autorisant ainsi la construction des bureaux du STCN, il faut attendre l'arrêté du 19 mars 1932 pour la remise des terrains le long de l'avenue. Cette cession permit l'édification de petits bâtiments en limite de parcelle, plus tard raccordés à l'aile est du STCN. Les principaux édifices élevés dans l'entre-deux-guerres trouvèrent place grâce à ces deux cessions. Quatre autres, consenties par la Ville en 1948, 1951, 1953 et 1954, autorisent l'extension vers l'ouest. Au sud, avant la construction du boulevard périphérique, se trouvait immédiatement le champ de manœuvres d'Issy-les-Moulineaux qui bridait les ambitions territoriales de la Marine, les services du ministère de l'Air, voisin, souhaitant y ouvrir un aérodrome.

21. Archives de Paris, permis de construire, VO ${ }^{12}$ 614. Le bâtiment est inauguré fin 1931, comme nous l'apprend une note de l'amiral Darlan sur les états de service de Camille Marion.

22. On lira avec profit l'analyse de Réjean Legault sur le caractère novateur du remplissage par panneaux de béton ainsi que la conception par Perret de l'immeuble de bureaux moderne. CULOT, Maurice, PEYCERÉ, David, RAGOT, Gilles (dir.). Les frères Perret. Paris: IFA, Norma, 2000, p. 183-187. 
23. Entre autres: DUPONT, H. Les nouveaux bâtiments du Service technique des constructions navales. La technique des travaux, juin 1932, n 6, p. 333-338.

24. Sur le rôle de Marion, voir infra note 27.

25. Le Conseil général des bâtiments de France demande une confirmation écrite de l'accord d'Auguste Perret, auteur de la composition initiale. L'architecte Camille Marion qui présente le projet répond qu'Auguste Perret est décédé... Si Gustave meurt en 1952, Auguste ne disparaît qu'en 1956 et c'est l'agence Perret qui réalise la surélévation. SHD/DM, 3DD ${ }^{2} 1099$. Et fonds Perret, AN/IFA, 535 AP 420/3.

26. COLLINS, Peter. Splendeur du béton. Paris : Hazan, 1995, p. 399.

27. Les états de service de Camille Marion (diplômé par le gouvernement, promotion 1911) nous renseignent sur la part exacte prise par ce dernier. Architecte de la Marine militaire (constructions navales) depuis 1926, il conduit et surveille les travaux exécutés par les frères Perret pour le STCN. C'est également comme architecte du ministère de la Guerre depuis 1912 qu'il exécute l'immeuble du Laboratoire central des poudres, quai Henri IV et comme architecte du ministère de la Marine militaire (artillerie navale) depuis 1913 qu'il exécute les bâtiments de ce service rue Sextius-Michel, tous travaux réalisés par l'entreprise Perret. Marion exécute également le bassin de giration du STCN. Il faut sans doute porter crédit à ses écrits, même si certaines sources ne mentionnent qu'un rôle de contrôle des travaux. En tout état de cause, le 26 novembre 1940, il est nommé architecte en chef de l'immeuble du STCN à titre provisoire, puis devient architecte en chef du gouvernement. À ces titres, il signe certains projets, comme la surélévation du bâtiment du STCN, mais aussi le canal lié au bassin de giration. Il exerce tantôt la fonction de maître d'œuvre, tantôt celle de maître d'ouvrage selon qu'il agit ou non comme architecte pour l'agence Perret. Sa part d'intervention est sans doute à réévaluer, notamment pour toutes les constructions d'après-guerre. Mais la qualité d'architecte conseil qu'il obtient si difficilement après-guerre sur plusieurs projets le place néanmoins davantage dans un rôle d'assistance que de conception. SHD/DM, 3DD 21099 et $\mathrm{F}^{21} 7865$ (personnel des monuments historiques des BCPN).

28. Une ambiguïté subsiste : Marion est chargé des projets de concours par convention signée le 20 octobre 1951 alors que le marché pour ces mêmes travaux, signalé sur un plan général du site, est daté du 28 août 1951... SHD/DM 3DD 2099.

29. AN/IFA fonds Perret, 535 AP 424/3.

30. COLLINS, Peter. Splendeur du béton. Paris : Hazan, 1995, p. 399.

31. http://www.defense.gouv.fr/defense/content/download/146309/1270197/file/dossier \% 20de \%20presse \%20projet \%20Balard \%20 \%20ACCORD \%20CABMIN \%2025 \%2003 \%2009.pdf

32. La création d'une annexe du bassin d'essai des carènes de Paris à Val-de-Reuil est décidée en 1981. À l'origine réservé à l'étude de la cavitation et d'écoulements, il est devenu le site définitif $\mathrm{du}$ bassin http://www.bassin.fr/. Il comprend un grand et un petit tunnel hydrodynamique, un grand bassin de traction $(600 \mathrm{~m})$, une cuve à houle, des ateliers, bureaux d'étude et services annexes. Il réalise des essais navals dans les domaines hydrodynamique et hydroacoustique pour des bâtiments de surface, des sous-marins et autres types d'engins. Le bassin est l'un des quatorze centres d'expertise et d'essai de la délégation générale pour l'armement (DGA) http:// www.defense.gouv.fr/dga/dossiers/essais_hydrodynamiques_et_hydroacoustiques

33. La question des instruments du bassin n'a pas été abordée mais mériterait d'être posée. La Jameson, machine anglaise qui réalisait la taille des maquettes en paraffine, est délocalisée à Valde-Reuil. Après l'interdiction d'utiliser la paraffine, elle fut reconvertie en "palpeur» pour vérifier la géométrie des maquettes aujourd'hui réalisée en fibre de verre stratifié avec armatures en bois ou bien totalement en lattes de bois. AUCHER, Max. 100 ans d'histoire du bassin d'essais des carènes. [s.l.] : DGA, Ministère de la Défense, [2006], p. 81 


\section{RÉSUMÉS}

Le «bassin des carènes" à Balard abrite un ensemble cohérent d'édifices techniques, scientifiques et administratifs destinés à l'étude du comportement des navires et de leur propulsion en toutes situations. Créé à l'initiative de l'ingénieur Bertin et inauguré en 1906, le premier bassin, en béton, comptait alors parmi les quatre plus grands bassins du monde. À partir de 1932, Perret frères édifient une véritable cité scientifique : bâtiment administratif (IMH 1965), laboratoires, plusieurs bassins sont élevés en béton armé, avec un même soin accordé à l'ensemble.

The 'bassin des carènes', the ship testing tank situated at Balard, in Paris, comprises a coherent ensemble of technical, scientific and administrative buildings devoted to the study of ships and their propulsion in different situations. The facility was first created in 1906 at the instigation of the engineer Bertin. At this date, the towing tank, constructed in reinforced concrete, was one of the four largest in the world. From 1932, the Perret brothers were commissioned to provide other constructions, completing an authentic scientific complex or 'city'. This included the administrative building (given historic monument protection in 1932) and several other tanks, all constructed in reinforced concrete and with the same attention to detail.

\section{INDEX}

Mots-clés : Auguste Perret, Balard, Bassin de giration, Bassin des carènes, Bassin d'essai, bastion, béton armé, Camille Marion, Canal, Cité de l'air, Frères Perret, Gustave Perret, Henri Chassin, Laboratoire, Louis-Émile Bertin, Maquette, Marine, Service technique des constructions navales, STCN

Keywords : Gyration test tank, Laboratory, Model, Navy, Perret brothers, Reinforced concrete, Service technique des constructions navales (STCN), Shipbuilding, Test tank, Towing test tank

\section{AUTEUR}

\section{VALÉRIE GAUDARD}

Chargée d'études, conservation régionale des monuments historiques, Direction régionale des affaires culturelles d'île-de-France. valerie.gaudard@culture.gouv.fr 